\title{
La justicia restaurativa como estrategia disciplinaria en el Centro Escolar Urbanización Bella Vista en el Departamento de Santa Ana en El Salvador ${ }^{1}$
}

Restorative justice as a disciplinary strategy at Centro Escolar Urbanización Bella Vista in the Department of Santa Ana en El Salvador

Miguel Ángel Pleitez Herrera ${ }^{2}$

Pleitez H, Miguel
miradas N²-2019 ISSN digital N²539-3812 Págs 146-162
Recepción: Febrero 12 de 2019
Aprobación: Junio 1 de 2019
Publicación: Junio 30 de 2019

\section{Resumen}

El objetivo de este estudio fue analizar la justicia restaurativa como estrategia disciplinaria en el Centro Escolar Urbanización Bella Vista en el Departamento de Santa Ana en El Salvador. Fue un estudio explicativo en el que se indaga la realidad disciplinaria de la institución, demostrando que esta era del tipo dictatorial. Además, en relación al aspecto antes mencionado; se indagaron los procedimientos disciplinarios y se descubrió que no se tenía claridad en su ejecución. Además, el desconocimiento de vías disciplinarias adecuadas para solventar conflictos, derivó que no se tuviera claro cuáles eran las tipologías de faltas y sanciones vertidas en el Manual de Convivencia Escolar, instrumento exigido por el Ministerio de Educación, Ciencia y Tecnología que de ahora en adelante se denominará (MINEDUCYT); así también, las funciones y agentes disciplinarios no estaban definidos de forma precisa. Por ello, se procedió a elaborar una propuesta partiendo en primer lugar de la realimentación de lo que establece el Manual de Convivencia Escolar, y dentro de eso, se anclaron procedimientos de corte restaurativo aplicados al contexto escolar. Sin duda el reto más grande será que exista una actitud positiva al cambio, ya que será una propuesta que se materializa en la convivencia de la comunidad educativa en estudio.

\footnotetext{
1 Este artículo es producto de los resultados obtenidos en el Trabajo de Postgrado presentado como requisito para optar al título de Máster en Asesoría Educativa, "LA JUSTICIA RESTAURATIVA COMO ESTRATEGIA DISCIPLINARIA EN EL CENTRO ESCOLAR URBANIZACIÓN BELLA VISTA EN EL DEPARTAMENTO DE SANTA ANA, EL SALVADOR”, en diciembre de 2018.

2 Máster en Asesoría Educativa. Productor de la Marca de Servicios Culturales Cuiloa Nu Tal... El Salvador (Adaptación de la frase Náhuatl Contar Historias de Mi Tierra El Salvador). Docente e Investigador. Actualmente desempeña el cargo de Técnico de Investigación en el Instituto Salvadoreño para la Protección Integral de la Niñez y la Adolescencia (ISNA).pleitezdante26@yahoo.com
} 
La justicia restaurativa como estrategia disciplinaria en el Centro Escolar Urbanización Bella Vista en el Departamento de Santa Ana en El Salvador

Palabras Claves: justicia restaurativa, manual de convivencia escolar, procedimiento disciplinario, tipos de disciplina, agentes disciplinarios, prácticas restaurativas

\section{Abstract}

The objective of this study was to analyze restorative justice as a disciplinary strategy at the Bella Vista Urbanization School Center in the Department of Santa Ana in El Salvador. It was an explanatory study in which the institution's disciplinary reality was investigated, demonstrating that it was of the dictatorial type. In addition, in relation to the aforementioned aspect; the disciplinary procedures were investigated and it was discovered that there was no clarity in its execution. In addition, the lack of knowledge of appropriate disciplinary ways to resolve conflicts led to a lack of clarity about the types of faults and sanctions included in the School Coexistence Manual, an instrument required by the Ministry of Education, Science and Technology; likewise, the functions and disciplinary agents were not defined precisely. Therefore, we proceeded to develop a proposal starting with the feedback of what is established in the School Coexistence Manual, and within that, restorative procedures applied to the school context were anchored. Undoubtedly the biggest challenge will be that there is a positive attitude to change, since it will be a proposal that will materialize in the coexistence of the educational community under study.

Keywords: restorative justice, school coexistence manual, disciplinary procedure, types of discipline, disciplinary

agents, restorative practices.

\section{Introducción}

En la actualidad, en El Salvador la práctica de valores que evoque una sana convivencia está siendo una preocupación latente, en este caso se toma en cuenta dentro de este marco, lo concerniente a la "disciplina escolar"; materializando su gestión a través del Manual de Convivencia Escolar; este instrumento escolar fue creado por autoridades del MINEDUCYT (Ministerio de Educación, Ciencia y Tecnología deElSalvador)en elaño 2007 con el propósito de ordenar y establecer los lineamientos fundamentales de las instituciones educativas en materia de prevención y resolución de conflictos de manera creativa, pacífica, justa y democrática; su elaboración toma en cuenta la participación de docentes, padres de familia, estudiantes y personal administrativo, con el fin de establecer las normas escolares y de convivencia que regulan el comportamiento de los miembros de la comunidad educativa.

Desde la implementación de este insumo se espera que las instituciones educativas los mantengan actualizados ante nuevas incorporaciones en materia legal respecto a la niñez y adolescencia. Ante este elemento el Maestro Carlos Canjura, Ministro de Educación, en una entrevista en mayo de 2018 planteó el lanzamiento de Política Nacional de Convivencia Escolar que busca, entre otras cosas, que se fomente el respeto y revisar que los centros de enseñanza públicos y privados tengan sus manuales de convivencia actualizados. 
El enfoque de Normas de Convivencia del instrumento antes citado, en teoría debe superar el carácter de los reglamentos internos y de sus normas disciplinarias dejando la sanción como un último recurso, dando prioridad a las respuestas pacíficas; ese aspecto formó parte esencial de la problemática investigada donde se detectaron las prácticas disciplinarias reales al interior de la comunidad educativa del Centro Escolar Urbanización Bella Vista, en el Departamento de Santa Ana; desde esos elementos se recomendó como alternativa de la temática, la "justicia restaurativa" la cual es un enfoque cimentado en la reparación de las relaciones en la comunidad, bajo un presupuesto de inclusión y respeto; aportando así al centro educativo una actualización concreta que mejorará las prácticas disciplinarias ejercidas.

La situación tomada como problemática, fue suscitada por el tipo de disciplina que se práctica al interior de la institución; la cual era de tipo dictatorío, donde las sanciones y procedimientos ante faltas cometidas, eran resueltas de forma punitiva.

Los sujetos de investigación afectados por esta problemática fueron los directivos, docentes, estudiantes y padres de familia del centro escolar, puesto que la manifestación práctica del proceder disciplinario es la convivencia escolar y al no realizarse correctamente, evita la construcción de un ambiente de paz y respeto, mostrado que las prácticas disciplinarias desarrolladas antes de la propuesta, no estaban dando los resultados adecuados. La institución en estudio atiende alrededor de 300 estudiantes en los turnos matutino y vespertino.

La convivencia escolar es un elemento de crucial importancia en el contexto educativo, pues plantea la dinámica relacional de los que ahí convergen; pero es preciso tener la noción de la misma para facilitar su comprensión. A este respecto Palomino y Paz (2010) plantean:

Convivir significa vivir unos con otros en base a unas determinadas relaciones sociales y a unos códigos, en el marco de un contexto social determinado donde los conflictos son inseparables por lo que no podemos pretender que las interacciones cotidianas $\mathrm{y}$ el proceso educativo transcurra sin conflictos, ni tampoco pensar que los mismos resultará necesariamente negativos. (p.87)

Aprender a conviviren un contexto como el escolar, requiere el adaptarse a normas propias de este, por ello es que surge la propuesta de justicia restaurativa, pues tradicionalmente la información y procedimientos de convivencia son vertidos en el Manual de Convivencia Escolar, los cuales como plantea el MINEDUCYT deben ser conocidos y cumplidos por la comunidad educativa, y el insumo sugerido; será de apoyo a ese instrumento.

La disciplina escolar, es un elemento importante que entra en juego en la 
dinámica de convivencia escolar, y según plantean Márquez, Díaz y Cazzato (2007) se define como "... el conjunto de normas que regulan la convivencia en la escuela" (p.127). Dentro de los procedimientos para el cumplimiento de las normas, se determina la justicia restaurativa como un insumo humanizado de los procesos, este enfoque como sostiene Rincón (2014) se define como

.. una estrategia
alternativa para la solución
de conflictos en el contexto
escolar, teniendo en cuenta
que el manejo tradicional
de la disciplina no ha sido
efectivo en las Instituciones,
puesto que los estudiantes
se han acostumbrado a ser
castigados o simplemente
a evadir su responsabilidad
a fin de evitar la sanción.
(p.19)

En esta perspectiva, se reconoce la participación de los diferentes actores que hacen parte de la comunidad educativa (Consejo Directivo Escolar) entendida esta como: estudiantes, docentes, directivos, docentes y padres de familia, lo que como sostiene Ruíz (2006) “...apuntar a los principios propuestos por la justicia restaurativa desde el diálogo, la mediación, la solución de conflictos y reparación de las víctimas" (p.54).

La justicia restaurativa según lo define Pérez y Huerta (2014) implica

...un proceso en el que las partes implicadas en un delito determinan de manera colectiva las formas en que habrán de manejar sus consecuencias e implicaciones. Representa una respuesta evolucionada al crimen, que respeta la dignidad y la equidad de cada persona, construyendo comprensión y promoviendo la armonía social, a través de un proceso de sanación de las víctimas, los ofensores y la comunidad. (p. 2)

La noción anterior, está enmarcada en un ámbito jurídico; espacio donde este enfoque surgió, en materia educativa Cortés (2012) la plantea como

...un cambio de posicionamiento y de mentalidad individual e institucional en relación con la gestión de las relaciones y de las situaciones de conflicto. Fundamentado en los valores del respeto mútuo, la responsabilización, la reparación y la resolución cooperativa, el enfoque quiere ser un motor de cambio, propiciar el abandono del sistema punitivo e incorporar nuevas metodologías de aula centradas en desarrollar, mantener $\mathrm{y}$ reparar relaciones de forma restaurativa. (p.3) 
La intencionalidad del enfoque restaurativo en ambas perspectivas coinciden, pues se busca una alternativa ante procedimientos disciplinarios punitivos que coarten la oportunidad de restaurar el tejido social. Con este enfoque se quiere reducir la solución de conflictos mediante mecanismos sancionatorios por una donde los involucrados puedan reparar lo dañado. Para ejercer este tipo de enfoque hay ciertos aspectos que se deben tener en cuenta, al respecto Pérez y Huerta sugieren:

a) La víctima y el ofensor deberán estar de acuerdo sobre su participación en los procesos y acuerdos restaurativos.

b) De no ser adecuada la aplicación del método restaurativo, el asunto deberá atenderse mediante la justicia penal.

La aplicación de un enfoque restaurativo no siempre funcionará, por eso se necesita la voluntariedad de los implicados y de no llegar a los acuerdos correspondientes, se procede a la aplicación de las normativas establecidas.

Se espera la implementación del diálogo restaurativo como herramienta de acercamiento al conflicto desde una visión comprensiva, en el que se escuchan las partes, se toman decisiones en conjunto y común acuerdo, en el que la norma no sea impositiva sino formativa y con base al consenso y beneficio de toda la comunidad escolar.

En términos generales con esta investigación se buscó responder a la siguiente pregunta: ¿Cómo implementar el enfoque de justicia restaurativa como alternativa disciplinaria en el Manual de Convivencia del Centro Escolar Urbanización Bella Vista en la Ciudad de Santa Ana?

Siendo las acciones a alcanzar con el proceso las siguientes: determinar el tipo de disciplina, identificar los procedimientos disciplinarios para la resolución de conflictos vertidos en el Manual de Convivencia y establecer una propuesta disciplinaria de justicia restaurativa encaminada a fortalecer la convivencia del Centro Escolar Urbanización Bella Vista desde el diálogo, la escucha activa y el afecto, como herramientas de acercamiento al conflicto desde una visión humana.

\section{Metodología}

Se realizó un estudio explicativo, con el cual se analizó la justicia restaurativa como estrategia disciplinaria en el Centro Escolar Urbanización Bella Vista en el Departamento de Santa Ana. Se realizó una caracterización, análisis e interpretación de la naturaleza actual de la disciplina en el centro escolar en estudio; además, de presentar una alternativa de disciplina escolar.

En cuanto a los Sujetos de Estudio los criterios de selección en relación con los sujetos de investigación fueron los siguientes:

I. Se ubica en una zona considerada de peligrosidad a nivel delincuencial, lo cual conlleva a que la propuesta, sea un aporte para mermar conductas negativas al interior del centro escolar, que pueden ser producto 
La justicia restaurativa como estrategia disciplinaria en el Centro Escolar Urbanización Bella Vista en el Departamento de Santa Ana en El Salvador

de las influencias violentas de la comunidad.

II. Otro criterio que se tuvo en cuenta fue que el centro escolar es relativamente nuevo en su funcionamiento, lo cual hace que una propuesta como la planteada, le permita la cohesión constructiva al contexto donde pertenece. Puesto que a la larga, si no existe una adaptación a la realidad; la comunidad educativa puede verse afectada con elementos externos que impidan su correcta administración o el óptimo desarrollo del proceso de enseñanza.

En total se seleccionó a nueve personas para realizar el estudio, dos directivos y siete docentes del Centro Escolar Urbanización Bella Vista.

En cuanto a las Técnicas e Instrumentos, se administró una entrevista estructurada, a través de un cuestionario abierto, para recoger sus apreciaciones y líneas de trabajo en la actualización de los procedimientos disciplinarios del manual de convivencia escolar. A los docentes se les aplicó una encuesta a través de un cuestionario cerrado, para detectar sus apreciaciones sobre los procedimientos disciplinarios y la dinámica interna del Manual de Convivencia Escolar.

También se realizó una búsqueda de información documental en el Manual de Convivencia Escolar del centro escolar, así como en manuales de justicia restaurativa, que permitieron estructurar la propuesta disciplinaria bajo el enfoque restaurativo, como un precedente para futuras acciones en el rubro de convivencia escolar de la comunidad.

En general, se realizó un procedimiento ordenado con las siguientes etapas: planificación, aplicación o trabajo de campo, procesamiento e interpretación de la información, análisis estadístico y socialización de la investigación.

\section{Resultados}

Los resultados del proceso investigativo se dividirán en dos partes: la primera será respecto a las generalidades relacionadas con la disciplina escolar y el procedimiento disciplinario; y la segunda, será que a partir de esos datos, se elaboró la propuesta disciplinaria de justicia restaurativa anclada al manual de convivencia escolar.

\section{A. De la disciplina escolar y el procedimiento disciplinario}

Con lo encontrado en el proceso investigativo en términos generales se puede establecer que el tipo de disciplina imperante en la institución educativa en estudio es: la dictatoría (los estudiantes saben lo que se espera de ellos pero se puede decir que en general ellos siguen las reglas por temor y también por no tener otra opción, más no por estar convencidos de que es lo mejor o lo más adecuado); con marcada tendencia hacia considerar como un modelo apropiado la disciplina ecuánime (son sinónimos de esta disciplina los siguientes: equilibrada, imparcial, justa, objetiva, íntegra. Este tipo de disciplina es reflexivo, porque busca un ejercicio disciplinario basado en la toma de decisiones, tanto 
para corregir las faltas así como para reflexionar sobre estas).

En relación con los procedimientos disciplinarios, se denota que no hay claridad en la gestión de la disciplina, lo que va desde el desconocimiento del reglamento y responsables del ejercicio de la disciplina; hasta el no tener definidas las instancias para la ejecución de un debido proceso, que permita al estudiante responder de forma justa y objetiva ante una falta cometida.

Lo anterior, fue base sin duda para plantear la propuesta disciplinaria con enfoque restaurativo, que hizo énfasis en la disciplina ecuánime y buscará fortalecer las nociones y procedimientos de la gestión disciplinaria en la institución educativa.

\section{B. Integración de la Estrategia de Justicia Restaurativa como parte del Manual de Convivencia Escolar}

Esta incorporación básicamente consiste en integrar al Manual de Convivencia Escolar de la institución educativa en estudio, procedimientos restaurativos, que replanteen la resolución de las faltas; mediante alternativas, que conlleven a brindar un proceso de orientación con base en el diálogo; logrando que la imposición de sanciones se reduzca, y no sea el único medio para tratar los conflictos de la realidad escolar.

El MINEDUCYT en sus Normativas de Funcionamiento Escolar, en relación con la disciplina escolar sostiene que lo establecido en el Manual de Convivencia Escolar no deben contradecir leyes primarias, secundarias, reglamentos $\mathrm{y}$ normativas que rigen al sistema educativo y la convivencia social; así como los tratados internacionales sobre derechos y deberes de niños y niñas, adolescentes y personas adultas.

Figura 1: Esquema Organizativo de la estrategia de Justicia Restaurativa

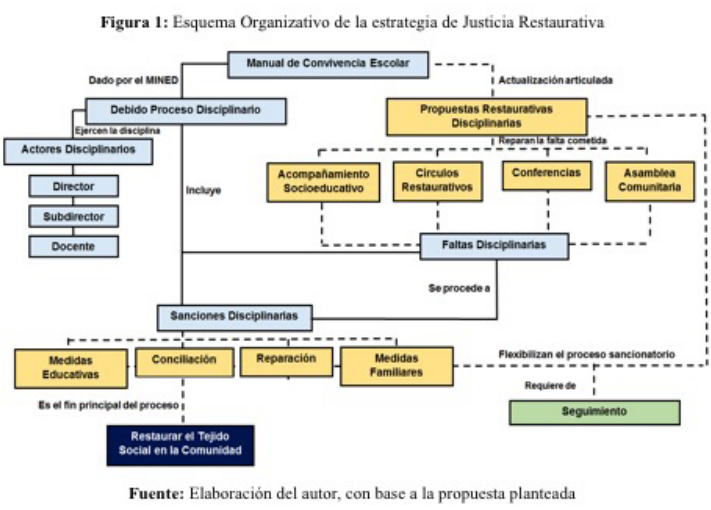

Partiendo de lo anterior, la incorporación debe adaptarse a todo lo que ya se estipula, en el marco disciplinario; respetando, ante todo, que lo sugerido no vaya en contra de los presupuestos legales del Sistema Educativo Nacional, ni que afecte de alguna manera la susceptibilidad de los actores que convergen en la comunidad educativa estudiada. A continuación se presenta el esquema organizativo de la propuesta planteada.

Este esquema muestra la dinámica de la propuesta con enfoque de justicia restaurativa, empleando la simbología siguiente:

1. Las líneas punteadas determinan las nuevas incorporaciones realizadas, incluyendo estas en color amarillo.

2. Las líneas sin puntuar, determinan lo que ya estaba contemplado dentro 
del manual de convivencia escolar, destacando esos elementos en color celeste.

3. Fuera de la estructura de incorporaciones se destaca en color verde, que esta propuesta debe estar bajo un proceso constante de seguimiento, para mejorar aquellos aspectos que lo requieran.

4. Se incluye en color azul el fin último de la propuesta el cual es que al aplicar todas las incorporaciones, se espera que más allá de una sanción, se logre restaurar el tejido dañado, fortaleciendo los lazos de convivencia de la comunidad.

\section{Nuevas incorporaciones de} Justicia Restaurativa al Manual de Convivencia Escolar

\section{Proceso de elaboración de normas de convivencia}

Las normas de convivencia deben ser conocidas por la comunidad educativa; y deben publicarse en todos los grados y secciones, mediante carteles, regulando el comportamiento y orden en los recintos escolares. (MINED, 2008)

Bajo la dinámica de esta propuesta, es importante reconocer; que una normativa de convivencia no debe ser punitiva ni coercitiva; se debe llegar al consenso de estas normas y tener claro el porqué se plantean.

Con el enfoque restaurativo, se trata de una nueva visión de la convivencia y disciplina en la escuela. Las normas de convivencia son pautas generales de comportamiento aplicables a la vida escolar, y comunes a todos los estudiantes. (IES, 2007)

Herrera (2007) sostiene aspectos básicos sobre los cuales se deben crear las normas de convivencia, atendiendo a la necesidad de cada aula:

I. Relaciones personales de los estudiantes entre sí y con los directivos, docentes, personal administrativo, padres de familia y demás adultos.

II. Actividad académica: Atención en clases que implica entre otros, el no distraer a los compañeros. Presentación de las tareas y trabajos a tiempo, estudiar para los exámenes, etc.

III. Salud e higiene: Cuidado de la salud consumiendo alimentos saludables, protegerse del cambio climático, no exponerse al frío si ha transpirado. Aseo personal, manos limpias, vestimenta limpia e idónea.

IV. Asistencia y puntualidad: No falta y llega a tiempo a la institución y a cada clase.

V. Cuidado del material propio, ajeno y de la institución. Cuida sus cosas y la de los demás. Pide permiso para usar lo que no es suyo. Trata con cuidado la infraestructura y mobiliario del aula.

A partir de lo anterior, se deben formular las normas de manera positiva, evitando la negación o imposición, se trata de destacar la razón de evocar el comportamiento de la norma y no solo imponer.

Las normas se formulan con enunciados propositivos, en primera persona del 
plural señalando el comportamiento deseado. Se recomienda no usar el deben. (Ejemplos: "Somos puntuales para llegar a la institución educativa y al entrar al aula después de los recreos porque así se puede desarrollar correctamente las clases", "Si necesito algo que no es mío le pido al dueño del objeto que me haga el favor de prestármelo porque no puedo tomar las cosas de otro sin permiso"). Además, estas deben ser breves y comprensibles por todos los integrantes de la comunidad educativa. (IES, 2007)

Planteando las normas de convivencia de esta forma, se logra el reconocimiento de las razones por que cumplirlas, permitiendo que no se transgredan; puesto, que adquieren un significado concreto, y no se limitan simplemente a coartar a los estudiantes. Además, estas normas deben ser presentadas a los padres de familia, para que se tenga claro; que al incumplirlas se incurrirá en una falta, que da lugar a un proceso disciplinario que puede llegar a ser sancionatorio.

\section{Acompañamiento socioeducativo}

Es una reformulación del concepto de tutoría; que se arraiga en un concepto central, clásico, de la pedagogía: el "mentor"; bajo este marco "orientar se llama acompañar" y en que esta es la única manera de influir en las vidas de los estudiantes. (Alonso y Funes, 2009)

Esta práctica, es transversal a todos los procesos restaurativos, ya que se busca que el docente acompañe, a los estudiantes con mayor necesidad conductual, en diversos momentos de la jornada escolar.

Este proceso, conlleva a una valoración del estudiante, explorando la personalidad del adolescente para obtener información sobre sus recursos personales, aspiraciones, necesidades $y$ otros aspectos que pueden ser incorporados en la construcción de un acompañamiento efectivo. (Salazar, 2012)

El acompañamiento es importante, ya que permite que se conozcan mejor a los estudiantes logrando una comunicación pertinente, para aplicar otro tipo de práctica disciplinaria.

\section{Conferencia (mediación reparadora)}

Aunque el término "mediación" fue adoptado durante los primeros años del desarrollo de la justicia restaurativa, se ha tendido a reemplazarlo cada vez más por palabras como "conferencia" o "diálogo". (Zehr, 2007)

Lo que hace que la conferencia difiera en sí, con el proceso de mediación, es en primera instancia el lenguaje empleado, pues en un proceso restaurativo el ofensor debe aceptar su falta; y sobre esa base se buscan alternativas se reparación; mientras que en una mediación se emplea un lenguaje neutral, limitando las responsabilidades de los implicados.

El autor antes citado, establece que en una mediación las partes están moralmente parejas, mientras en una restauración cada parte, reconoce su implicancia en el conflicto; sin eso es 
La justicia restaurativa como estrategia disciplinaria en el Centro Escolar Urbanización Bella Vista en el

Departamento de Santa Ana en EI Salvador

imposible ejecutar prácticas de este tipo.

En relación con la ejecución de este proceso Salazar (2012) plantea que hay dos formas de realizar las conferencias (mediación reparadora), las cuales se exponen en la Figura 2:

Figura 2. Tipos de Conferencias (mediación reparadora)

Conferencia Directa: es aquella

utilizada para reparar el daño,

cara a cara; entre la victima, el

ofensor y un tercero llamado "mediador".

Conferencia Indirecta: es aquella utilizada para reparar el daño sin que necesariamente se lleve a cabo el encuentro entre las partes (ofensor o victima). Tambienn participa el mediador.

Fuente: Caja de Herramientas. Justicia Juvenil Restaurativa. Salazar (2012

El mediador, en este plano es aquella figura que su función principal es informar, explorar, valorar, preparar y conducir el proceso de encuentro con neutralidad, lo que legitimara los compromisos a los que lleguen las partes. Deberá propiciar el respeto mutuo entre las partes, así como la escucha activa. De ninguna manera podrá proponer fórmulas de solución. (Salazar, 2012)

Este proceso se propone realizar, en faltas leves donde la afectación de la comunidad sea mínima, ya que puede no llegar hasta la reunión de las partes tampoco requiere que haya más miembros de la comunidad para ejecutarse; pues con un mediador asertivo se pueden reparar los problemas. La reparación o restitución en este contexto de mediación es la contribución que puede realizar la persona infractora compensando en lo posible el daño causado a la víctima. (Gorbeña y Romera, 2008)

Si en caso la aplicación de esta práctica no resulta efectiva; se procede a documentar lo ocurrido y aplicar otro tipo de práctica que permita la resolución sana de lo ocurrido.

\section{Círculos restaurativos}

El uso del círculo restaurativo se usó en muchas comunidades aborígenes en Canadá. En este tipo de práctica todos los participantes, se sientan frente a los demás en un círculo. Los dictámenes en esta modalidad están generalmente disponibles solamente para aquellos delincuentes que se declaran culpables.

Las discusiones en el círculo están diseñadas para llegar a un consenso sobre la mejor manera de resolver el conflicto y disponer el caso, tomando en cuenta la necesidad de proteger a la comunidad, las necesidades de las víctimas y la rehabilitación y castigo del ofensor. (Oficina de las Naciones Unidas, 2006)

Dentro de la dinámica en estudio, esta práctica puede emplearse en la ocurrencia de faltas de tipo moderada a grave; puesto que estas ya incurran de manera directa en el bienestar de otras personas, y por ende de la comunidad.

Lo antes planteado, solo será efectivo si dentro de la realización, se llega a acuerdos que beneficien a las partes, y que haya sido un consenso de ellos, y no de ningún agente externo al problema (directivo, docente o compañero); de lo contrario se procede a documentar lo acontecido en el círculo, como base para 
la implementación de otra estrategia.

Aunque no existe una manera estándar o una receta para la consecución de los círculos Mata (2016) plantea ciertos elementos a considerar para su realización, que se describen en la Figura 3:

\section{Figura 3. Elementos que considerar para} realizar Círculos Restaurativos
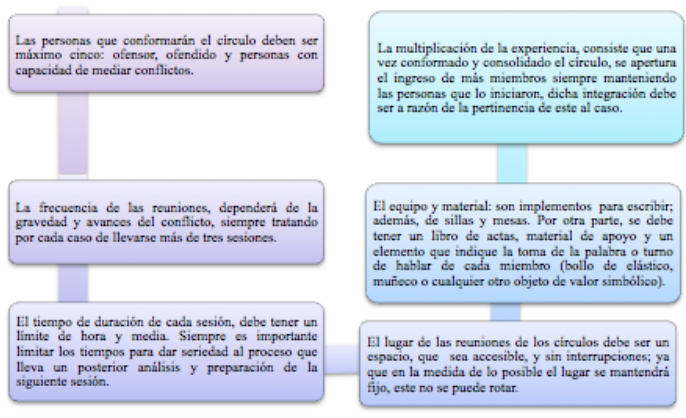

\section{Asamblea Comunitaria}

La asamblea comunitaria tiene un planteamiento más amplio que el de las conferencias o los círculos, ya que implica la participación de un mayor número de miembros de la comunidad educativa en la que se ha producido la infracción.

Puede ser considerada una forma expandida de la conferencia, que, de forma flexible, proporciona un foro a las personas participantes para encontrar sus propias soluciones ante una conducta que ha causado un daño. (Gorbeña y Romera, 2008)

Dentro de este proceso, se incorporan a los responsables de los implicados; porque se reconoce la importancia de la familia en la vida de cualquier persona y la especial necesidad y apoyo que ésta requiera cuando un miembro de esta ha cometido una infracción.

Esta práctica se da ante faltas graves, donde es necesario involucrar completamente a la comunidad educativa para llegar a un punto de reparación de todos los implicados; en caso de que esta práctica no funcione se procede a documentar lo ocurrido y pasar al debido proceso disciplinario de la institución educativa.

\section{Debido proceso disciplinario según Manual de Convivencia Escolar}

Puntualizando respecto a este proceso, se enuncian aquellos mecanismos a realizarse para la aplicación de disciplina en contexto escolar (Consejo Directivo Arturo Prat, 2018):

I. Que el ofensor sea tratado con el debido respeto en relación con su dignidad humana.

II. Que la familia sea informada mediante citación escrita o llamado telefónico, sobre la situación que corresponde a su hijo (académica, disciplinaria), así como las acciones a seguir y sobre los derechos que tiene.

III. Que la decisión para ejecutar el proceso esté precedida de un proceso de acompañamiento personal (docente) mediante el cual se indaguen las razones que llevaron al estudiante a transgredir las normas ${ }^{3}$.

3 Según el apartado anterior, en el marco de la propuesta disciplinaria de justicia restaurativa; solo se dará apertura al debido proceso disciplinario, en primer lugar, si la 
La justicia restaurativa como estrategia disciplinaria en el Centro Escolar Urbanización Bella Vista en el Departamento de Santa Ana en El Salvador

IV. Que el estudiante sea escuchado para hacer sus respectivos descargos con relación a las faltas cometidas, quedando registro escrito.

V. Que los padres y/o apoderados sean notificados oportunamente sobre la situación del estudiante, y la posible sanción de la falta cometida, según la gravedad de esta.

VI. Dejar un acta o registro de citaciones y entrevistas; así como del debido proceso disciplinario realizado.

VII. Que el estudiante sancionado puede manifestar su apelación de forma oral o escrita.

VIII. Todo formato de informe de seguimiento disciplinario se anexará al currículo del estudiante y constituirá antecedentes para la imposición de sanciones disciplinarias en su contra.

Este proceso, de carácter punitivo; media vez de comienzo, limita la reconstrucción de lo dañado, encontrando en una sanción, la solución al problema. Pero, así como se ha propuesto qué hacer antes de llegar a estas circunstancias, también en el siguiente apartado se plantean acciones, que apoyadas a este proceso, pueden orientar al ofensor a la reparación de su falta.

falta transgrede alguna ley vigente en materia de la niñez y adolescencia; y, en segundo lugar, cuando después de efectuar medidas restaurativas previas, estas no funcionen.

\section{Prácticas por implementar en el debido proceso disciplinario}

Se espera que las prácticas empleadas, durante el debido proceso disciplinario, no solo queden plasmadas en un expediente estudiantil; sino que sea reconocida por el ofensor y se intente (según la voluntariedad de los que convergen en el proceso) subsanar lo ocurrido: todo con el fin que la comunidad no sufra una fractura social, a razón de una sanción fríamente colocada.

\section{a) Medidas Educativas}

Siempre que se produzca una falta disciplinaria, antes de iniciar el debido proceso, el docente deberá intentar corregirla mediante prácticas disciplinarias; siempre y cuando se cuente con la aceptación del estudiante y de sus padres. Si estas prácticas funcionan, se determinará que no se inicie el procedimiento disciplinario y que tampoco se coloque una sanción; esto significa que no se podrá en marcha la gestión administrativa del debido proceso sancionatorio ${ }^{4}$. Entre estas prácticas están: acompañamiento socioeducativo, círculos restaurativos, conferencias (mediación restaurativa) $y$ asamblea comunitaria ${ }^{5}$

\section{b) Conciliación}

Una vez iniciado el debido proceso disciplinario, se produce la conciliación entre el estudiante ofensor y la víctima,

4 De acá en adelante, cuando se haga referencia a detener o proceder con el procedimiento disciplinario, se hará referencia a este aspecto.

5 Véase apartado "Prácticas restaurativas en el Ámbito Educativo" en la página 56. 
se suspende cualquier otro tipo de procedimiento en marcha.

La conciliación implica el reconocimiento de las consecuencias de la conducta, la presentación de disculpas o excusas, la aceptación de estas por parte de la persona ofendida o por el órgano correspondiente y la aceptación de la realización de alguna actividad educativa (Gorbeña y Romera, 2008). Aclarando, que esta conciliación se ha dado a partir de las partes implicadas, y no por sugerencia del docente, directivo o padre de familia.

\section{c) Reparación}

Esta acción es posterior a la colocación de la sanción puesto que nada de lo previamente ejecutado, funcionó. Salvo algunos casos como sostiene Gorbeña y Romera (2008) se suspende el procedimiento iniciado, si durante el transcurso, ya se ha producido la reparación; y esto ha sido asumido por el ofensor como un compromiso voluntario.

\section{d) Medidas Familiares}

Dentro de los mecanismos para potenciar el carácter educativo, se incluye la posibilidad de asociar el entorno familiar ${ }^{6}$ al cumplimiento de las medidas correctoras posterior al proceso disciplinario.

Abriéndose la posibilidad, de que se puede suspender alguna sanción; siempre y cuando la conducta del estudiante haya sido o esté siendo corregida en el ámbito familiar de 6 Se refiere al hecho de que se convocarán a los padres a participar del proceso, asistiendo a reuniones programadas o ejecutando acciones de reparación según sea el caso. manera adecuada, ajuicio de la dirección del centro. (Gorbeña y Romera, 2008)

Todas las prácticas descritas, deben documentarse para analizar posteriormente lo logrado a partir de éstas. Con lo planteado se busca que el debido proceso disciplinario pueda ser flexible y sistemático; con la posibilidad de detenerlo según sean las circunstancias del conflicto. Aunque exista una sanción, siempre se brinde la apertura a reparar lo ocurrido; para que el estudiante visualice que el proceso busca reconstruir el tejido dañado y no afectar únicamente el expediente académico-conductual de este.

\section{Discusión}

En todo cambio, siempre surgen miedos y apatías de los que lo vivencian; eso si persiste puede entorpecer la fluidez del proceso; por eso, si se quiere articular es preciso alcanzar los siguientes aspectos:

A. Que la comunidad educativa se motive en la ejecución de esta propuesta (no con una visión administrativa, sino humana del proceso) donde cualquier mejora hecha en equipo sea de beneficio colectivo.

B. La eficacia de estas alternativas disciplinarias depende, en gran medida, de la participación directa y del compromiso de todas las personas implicadas en la gestión de la disciplina; dejar de lado que solamente los directivos son los que deben ser los encargados de estos procesos. 
La justicia restaurativa como estrategia disciplinaria en el Centro Escolar Urbanización Bella Vista en el

C. Que en la institución educativa existan espacios para socializar mecanismos disciplinarios, destacando los actores e instancias de estos. Con claridad en esos aspectos, se puede aplicar correctamente un enfoque restaurador.

D. Es necesario el ordenamiento de los actores con sus obligaciones en materia disciplinaria; ya que cada ente dentro de la comunidad educativa tiene sus funciones. Respetarlas genera orden y un clima óptimo para disciplinar.

E. Gestar la necesidad de mejora constante y de actualización docente; es como se puede ejecutar cualquier propuesta con vías al éxito.

F. Se pretende disminuir las situaciones de conflicto en torno a la comunidad educativa, involucrar las familias al proceso de convivencia escolar y generar un clima escolar favorable; pero eso será posible, si los que serán parte de la propuesta no ponen de su parte. La actitud es clave para ejecutar cambios.

Para el manejo alternativo de situaciones de conflicto, se hace necesario involucrar a docentes y padres de familia, apostándole a un cambio de mentalidad, porque es justo eso lo que implica darle un manejo formativo al cumplimiento de las normas. Por ello, es preciso empoderar en primera instancia a docentes y directivos, porque implementar la Justicia Restaurativa en el ámbito escolar, implica romper esquemas no sólo al interior de la institución educativa, sino también a nivel comunitario.

Conclusiones

Partiendo del cuestionamiento base de este estudio, relacionado con la implementación de la Justicia Restaurativa en el marco disciplinario del Centro Escolar Urbanización Bella Vista, se puede plantear que efectivamente, este enfoque disciplinario será un aporte en la prevención y mitigación en situaciones de conflicto en la realidad escolar, puesto que brinda herramientas para la resolución de problemas disciplinarios con una postura crítica y humana, forjando prácticas sanas de resolución de faltas basadas en el diálogo y la concertación.

En términos generales, tras la consecución de este estudio, se obtuvo información, resultados y propuestas; que llevaron a que se concluye lo siguiente:

La disciplina escolar, es sin duda un eje articulador que sienta las bases de la convivencia en la comunidad. En el estudio se encontró que el desconocimiento de una estructura disciplinaria clara y definida, evoca en prácticas dictatoriales que se traducen en la resolución de conflictos de una manera impuesta y punitiva, que limita la capacidad de gestar relaciones de convivencia sanas, entre los que incurren en una falta. Ante esto Rodríguez (2015) sostiene que procesos disciplinarios punitivos, limitan la reflexión de quien comete la falta, ya que la regulación es externa y las normas son impuestas, independientemente de esas imposiciones sean asertivas o erráticas, teniendo como producto únicamente un castigo (sanción). 
La normativa disciplinaria, que se concretiza en los procedimientos disciplinarios, debe ser estudiada constantemente mediante los seguimientos correspondientes, determinado qué aspectos pueden cambiarse en relación a esta; asimismo la comunidad educativa debe participar de esos procesos, y de la socialización de los mismos. Pues todo desconocimiento, provocará que ante determinado caso, no se le brinde la cobertura disciplinaria asertiva y coherente. Domínguez (2010) plantea al respecto, que la institución educativa en su conjunto debe luchar por conseguir la integración de la comunidad y hacerla partícipe de los procesos formativos de los estudiantes.

La implementación del enfoque de justicia restaurativa en el ámbito escolar proporciona elementos, que como sostiene Delors (1997) permitan que se aprenda a convivir; mediante el estímulo de la participación, la solidaridad, el diálogo, la responsabilidad y la autonomía, lo cual será la base para formar sujetos críticos con conciencia de cambio que contribuyan a la construcción de la democracia dentro de la realidad en la que se encuentran.

Dentro del proceso investigativo se pudo visualizar, que para plantear la propuesta de justicia restaurativa: en primer lugar, se debe ordenar el proceso disciplinario que el MINED establece a nivel institucional, dada las carencias en su ejecución de la comunidad en estudio; y luego a partir de esto, se articula la propuesta, que no busca cambiar lo que el Sistema Educativo Nacional reglamenta, más bien se espera flexibilizar y concientizar sobre cómo se está desarrollando la convivencia, en relación al perdón, la reparación y la restauración de las faltas cometidas. Constituyendo un panorama, como propone Zapata (2008) que sustenta el postulado: “...que es necesario el cambio de lente en el modo de concebir este tipo de situaciones disciplinarias, para que de manera expresa la justicia restaurativa pueda tener cabida en el ámbito escolar."

En definitiva, todo lo expuesto en este estudio, pretende que desde la convivencia escolar, columna vertebral de toda institución educativa; se piense el papel de lo formativo y restaurativo, a la hora de resolver problemas relacionados con la disciplina; construyendo así, herramientas y procesos reflexivos que involucren a directivos, docentes y estudiantes en la implementación de medidas dinámicas que tengan como eje la humanización de las relaciones en el ámbito escolar.

Es importante destacar, que son necesarias futuras investigaciones que abarquen este tema, debido a que esta temática no ha sido tratada con detenimiento en estudios anteriores, en este contexto. A partir de lo realizado se pueden dejar como futuras líneas de investigación las siguientes:

A. ¿Qué relación existe entre la novedad de la institución educativa en el contexto con su carencias en el manejo de procesos disciplinarios propuestos por el MINED?

B. ¿Por qué la comunidad educativa en estudio no tiene una base de lo que el MINED establece en materia disciplinaria? 
La justicia restaurativa como estrategia disciplinaria en el Centro Escolar Urbanización Bella Vista en el

Departamento de Santa Ana en El Salvador

C. ¿Existe algún tipo de limitante a nivel gerencial y docente que esté afectando la implementación de actualizaciones en diversa vías?

D. ¿Por qué existe una falta de liderazgo de parte de la comunidad educativa en relación a la disciplina $\mathrm{y}$ otros aspectos relacionados con los procesos formativos de los estudiantes?

E. ¿Qué tipo de comunicación y relaciones se ejerce en la comunidad educativa?

Seguir avanzando en este tipo de acciones hará que se fortalezca el quehacer educativo con miras a formar ciudadanos capaces de transformar la realidad social que en El Salvador acontece.

\section{Referencias Bibliográficas}

Alonso,I.yFunes, J.(2009). ElAcompañamiento en los Recursos Socioeducativos. Revista de Intervención Educativa, 28-46.

Antonio, R. (2014). Guía Metodológica para la Implementación de Prácticas Restaurativas en Municipios de Mejicanos, Ayutuxtepeque y Cuscatancingo. San Salvador: SSPAS.

Canjura, C. (29 de mayo de 2018). Educación revisará reglamentos de escuelas y colegios de El Salvador. (S. Joma, Entrevistador)

Cortés, M. (2012). Hacia Una Escuela Justa: de la Incorporación de la Justicia Restaurativa en el Ámbito Escolar. Parlem de Pedagogía, 1-22.

Delors, J. (1997). La educación encierra un tesoro: Informe a la UNESCO de la Comisión Internacional sobre la Educación para el Siglo XXI. México: Correo de la UNESCO.

Gladis, M. (2007). La Disciplina Escolar. Sinaloa: Sinaloa.
Gorbeña, L. y Romera, C. (2008). Prácticas Restaurativas en el Ámbito Escolar y Comunitario. España: Geuz.

Guía Pedagógica para la Convivencia Escolar. (2014). Bogotá.

Henríquez, U., Lancheros, D. y León, C. (2013). Cartilla de Fundamento para Educadores de Justicia Restaurativa. Arcila.

Herrera, R. (2007). Normas de Convivencia. Andalucía: Thecnograpic.

Howard, Z. (2007). El Pequeño Libro de Justicia Restaurativa. Estados Unidos de América: Good Books.

IES, N. (2007). Pautas para Elaborar Normas de Convivencia. Madrid.

López, C. y Pérez, S. (2016). Análisis Comparativo de la Implementación Metodológica del "Manual de Participación Estudiantil para el Fortalecimiento de la Convivencia Escolar", San Salvador.

Márquez, J., Díaz, J. y Cazzato, S. (2007). La disciplina escolar: aportes de las teorías. Revista de Artes y Humanidades, UNICA, 126148.

Martín, M., Buquest, C. y Villafuerte, R. (2012). La disciplina escolar desde un enfoque psicoeducativo para promover la intervención efectiva del profesorado. Revista Digital del Centro de Profesorado Cuevas - Olulas (Almeria), 65-77.

Mata, J. (2016). Prácticas Restaurativas: "Un Nuevo Enfoque De Trabajo Con Las Personas Imputadas y La Sociedad Civil Que Desea Generar Verdaderos Cambios Sociales Para Minimizar Los Altos Índices De Violencia Que Afronta La Sociedad Salvadoreña". San Salvador.

Ministerio de Educación (2018). Convivencia Escolar. Obtenido de Convivencia Escolar. Recuperado de: http://www.convivenciaescolar. $\mathrm{cl} /$ index2.php?id_seccion $=\quad$ portal $=50 \& \mathrm{id}$ contenido $=1791 \overline{6}$ 
Ministerio de Educación (2007). Paso a paso en la elaboración de manual de convivencia escolar. San Salvador.

Moderno, D. (2015). Manual de Convivencia Gimnasio Moderno. Bogotá.

Monarca, H. (2013). Aportes de Investigaciones sobre Transiciones Escolares. REOP, 116-125.

Mondaca, R. (2015). Convivencia y Conflictividad Escolar. Chile.

Oficina de las Naciones Unidas, c. 1. (2006). Manual Sobre Programas de Justicia Restaurativa. Viena: ONU.

Oviedo, M., y Tocaín, F. (2014). Procedimiento Disciplinario. Uruguay.

Palomino, M. y Paz, A. (2010). Los Problemas de Convivencia Escolar: Percepciones, Factores y Abordaje en el Aula. Suplemento Memorias V Encuentro, 87.

Patiño, D. y Ruíz, A. (2015). La justicia restaurativa: un modelo comunitarista de resolución de conflictos. Revista Facultad de Derecho y Ciencias Políticas, 2013-2055.

Pérez, J. y Huerta, J. (2014). Justicia Restaurativa: del Castigo a la Reparación. México.

Prat, E. D. (2018). Manual de Convivencia Escolar. Temuco.

Rincón, H. (2014). Gestión de Mejora de la Convivencia Escolar desde la Parámetros de Justicia Restaurativa. Medellín: Medellín.

Rodríguez, J. (2015). Programa de Disciplina Positiva. EJES, 27-32.

Ruiz, C. (2005). Estrategias de Aprendizaje. Caracas: Bolivariano.

Ruíz, R. (2006). La Convivencia Escolar: qué es y cómo abordarla. Andalucía: Novograf.

Salazar, O. (2012). Caja de Herramientas. Justicia Juvenil Restaurativa. Perú: Chataro Editores.
Salvador, M. (2008). Normativa de Funcionamiento. San Salvador: Imprenta Nacional.

López, L. (2014). Convivencia Escolar. Apuntes Educación y Desarrollo 2015, 1-17.

Villafuerte, R. (2010). Disciplina Escolar: Desarrollo y Aplicación de un Programa Actitudinal-Cognitivo para la Formación Permanente del Profesorado de Educación Primaria. Barcelona.

Zapata, G. (2008). Estrategia pedagógica desde la Justicia Restaurativa: Un estudio de caso en la Institución Educativa Javiera LondoñoCentro. Antioquia. 